\title{
Top mass and classification of heterotic superstring vacua
}

\section{J. Rizos*}

Physics Department, Theory Division, University of Ioannina

E-mail: irizosduoi.qr

We consider a class of $Z_{2} \times Z_{2}$ heterotic superstring vacua, characterised by $S O(10)$ gauge symmetry broken to the Pati-Salam gauge group, $S U(4) \times S U(2)_{L} \times S U(2)_{R}$, by the use of a discrete Wilson line. A series of criteria is employed in order to select phenomenologically interesting vacua. These include the presence of symmetry breaking Higgs fields, the absence of exotic matter and the presence of top quark mass coupling in the tri-level superpotential. A concrete exemplary model that satisfies all criteria is presented and analysed in detail.

Proceedings of the Corfu Summer Institute 2011 School and Workshops on Elementary Particle Physics and Gravity

September 4-18, 2011

Corfu, Greece

\footnotetext{
* Speaker.
} 


\section{Introduction}

String theory provides the framework for a unified description of all forces including gravity. However, in four dimensions, the theory possesses a huge number of vacua, the so called 'landscape' [W]. In the limit of low energies, a subset of these vacua is expected to lead to some effective field theory that reproduces the basic phenomenological features of the Standard Model of gauge interactions. In the absence of a stringy vacuum selection mechanism, a lot of effort has been put into the study of general properties and classification of these vacua in various formulations [回, 目, 田, 回, 目].

In reference [可] a method has been developed that allows for a detailed analysis of a big number of superstring vacua in the Free Fermionic Formulation [ [] of the weakly coupled heterotic superstring. This approach is guided by low energy phenomenology starting from the fact that the Minimal Supersymmetric Standard Model (MSSM) fermion generations together with the righthanded neutrino, fit perfectly into the spinorial representations of an $S O(10)$ gauge factor, while the vectorials of the same group can accommodate the MSSM breaking Higgs particles. The authors have identified an interesting subclass of superstring vacua with $S O(10)$ gauge symmetry and derived analytic formulae regarding the number of spinorial/antispinorial and vectorial representations. Furthermore, the $S O(10)$ gauge symmetry can be broken at the string level to a subgroup of $S O(10)$ that includes the MSSM. The simplest choice, from the technical point of view, is the PatiSalam (PS) gauge symmetry [ [8, Q]. The main phenomenological characteristics of each model, as the number of fermion generations, the number of candidate Higgs doublets and the number of exotic matter representations can also be calculated analytically. Computer search is then used to classify the PS vacua and a series of phenomenological criteria can be imposed on the spectrum in order to identify models compatible with the MSSM al low energies. Typical criteria are: three fermion generations, the existence of Pati-Salam breaking Higgs multiplets, the existence of $S U(2)_{L}$ Higgs doublets, the absence of exotics.

Implementation of the method provides some interesting results in the case of the Pati-Salam supertring vacua, comprising approximately $10^{15}$ models [ए人]. A big number of models, of the order of $10^{9}$, satisfy all phenomenological constraints, including the absence of massless exotic fractionally charged matter. This is a rather surprising result given the fact that the existence of fractional charged exotics is a generic property of Standard Model string vacua [ए]]. Actually the "exophobic" models presented in [12] where, to our knowledge, the first semi-realistic models where all exotics are massive at the string level.

Reducing further the set of acceptable models, requires extra phenomenological constraints involving couplings. For example, an important selection criterion is the existence of the top quark mass coupling at the tri-level superpotential. Effective implementation of this constraint, involves the analytic evaluation of the associated correlation function, for a generic model in the class under consideration and significantly reduces the number of acceptable models to a few million [[13]. Removing redundancies, that are quite generic in these constructions, we end up with a few hundred distinct models.

Our classification method has been particularly fruitful in identifying "islands" of phenomenologically attractive models in the heterotic "landscape". Nevertheless, a much more elaborate procedure is required before deciding whether a model could be qualified as (semi)realistic and a lot of 
things could go wrong at the various stages of the analysis. A specific model, that meets all criteria, has been constructed and analysed in [14]], explicitly demonstrating that models in this class are of particular phenomenological interest.

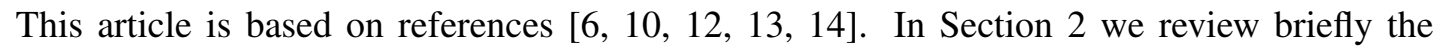
classification method and the set of phenomenological criteria for model selection. In Section 3 we review the analysis of an exemplary model.

\section{Classification method and Phenomenological constraints}

A heterotic string model in the Free Fermionic Formulation [ [ $]$ is defined in terms of a set of $n$ basis vectors

$$
B=\left\{v_{1}, v_{2}, \ldots, v_{n}\right\}
$$

and a set of $n(n-1) / 2$ phases, associated by the generalised GSO projections (GGSO), denoted by

$$
c_{i j}=c\left[\begin{array}{c}
v_{i} \\
v_{j}
\end{array}\right], i>j=1, \ldots, n
$$

subject to some constraints that guarantee modular invariance. Each basis vector consists of a set of phases $v_{i}=\left\{a_{i}\left(f_{1}\right), a_{i}\left(f_{2}\right), \ldots\right\}$ that describe the transformation properties of fermionised degrees of freedom. In the light cone gauge these are : 20 left fermions $\left\{\psi^{\mu}, \chi^{1, \ldots, 6}, y^{1, \ldots, 6}, \omega^{1, \ldots, 6}\right\}$ and the 44 right ones $\left\{\bar{y}^{1, \ldots, 6}, \bar{\omega}^{1, \ldots, 6}, \bar{\eta}^{1,2,3}, \bar{\psi}^{1, \ldots, 5}, \bar{\phi}^{1, \ldots, 8}\right\}$.

In [ㅈ] a phenomenologically interesting class of models, with Pati-Salam gauge symmetry, has been considered. This class is generated by the following set of 13 real basis vectors

$$
\begin{aligned}
& v_{1}=1=\left\{\psi^{\mu}, \chi^{1, \ldots, 6}, y^{1, \ldots, 6}, \omega^{1, \ldots, 6} \mid \bar{y}^{1, \ldots, 6}, \bar{\omega}^{1, \ldots, 6}, \bar{\eta}^{1,2,3}, \bar{\psi}^{1, \ldots, 5}, \bar{\phi}^{1, \ldots, 8}\right\} \\
& v_{2}=S=\left\{\psi^{\mu}, \chi^{1, \ldots, 6}\right\} \\
& \text { shifts }: v_{2+i}=e_{i}=\left\{y^{i}, \omega^{i} \mid \bar{y}^{i}, \bar{\omega}^{i}\right\}, i=1, \ldots, 6 \\
& Z_{2} \text { twist }: v_{9}=b_{1}=\left\{\chi^{34}, \chi^{56}, y^{34}, y^{56} \mid \bar{y}^{34}, \bar{y}^{56}, \bar{\eta}^{1}, \bar{\psi}^{1, \ldots, 5}\right\} \\
& Z_{2} \text { twist }: v_{10}=b_{2}=\left\{\chi^{12}, \chi^{56}, y^{12}, y^{56} \mid \bar{y}^{12}, \bar{y}^{56}, \bar{\eta}^{2}, \bar{\psi}^{1, \ldots, 5}\right\} \\
& v_{11}=z_{1}=\left\{\bar{\phi}^{1, \ldots, 4}\right\} \\
& v_{12}=z_{2}=\left\{\bar{\phi}^{5, \ldots, 8}\right\} \\
& v_{13}=\alpha=\left\{\bar{\phi}^{4,5} \bar{\phi}^{1,2}\right\}
\end{aligned}
$$

Vector $v_{1}=1$ is necessary for consistency, $v_{2}=S$ is required by $N=1$ space-time supersymmetry, $v_{9}=b_{1}, v_{10}=b_{2}$ correspond to the $Z_{2} \times Z_{2}$ ordifold twists and $v_{3}, \ldots, v_{8}$ are orbifold shifts. Vectors $v_{1}, \ldots, v_{12}$ give rise to $S O(10) \times U(1)^{3} \times S O(8)^{2}$ gauge symmetry while $b_{13}$ breaks the gauge symmetry to

$$
G=S U(4) \times S U(2)_{L} \times S U(2)_{R} \times U(1)^{3} \times S U(2)^{4} \times S O(8)
$$

In the following we will interpret $S U(4) \times S U(2)_{L} \times S U(2)_{R} \times U(1)^{3}$ as the "observable" and $\times S U(2)^{4} \times S O(8)$ as the "hidden" sector of the theory. In this class we have $13(13-1) / 2=78$ 
independent real GGSO coefficients $c\left[\begin{array}{l}v_{i} \\ v_{i}\end{array}\right]= \pm 1, i>j=1, \ldots, 13$. Restricting to $N=1$ supersymmetric models and eliminating some obvious redundancies we are left with 51 independent GGSO phases. Each coefficient can take values \pm 1 and a complete set of these phases defines a model, therefore this class a priory comprises $2^{51} \sim 10^{15}$ configurations. An advantage of this construction, at the technical level, is that the gauge group is determined primarily from the basis vectors and thus is fixed, while certain enhancements that appear for particular choices of the GGSO phases can be easily eliminated.

Another advantage of this formulation is the separation of matter spectrum in different sectors, controllable by GGSO projections:

- States belonging to $S O(10)$ spinorials not charged under $S U(2)^{4} \times S O(8)$. These belong to $\mathbf{1 6}, \overline{\mathbf{1 6}}$, truncated by the GGSO projections, $S O(10) \supset S U(4) \times S U(2)_{L} \times S U(2)_{R}$,

$$
\begin{aligned}
& \mathbf{1 6}=(\mathbf{4}, \mathbf{2}, \mathbf{1})+(\overline{\mathbf{4}}, \mathbf{1}, \mathbf{2}) \\
& \overline{\mathbf{1 6}}=(\overline{\mathbf{4}}, \mathbf{2}, \mathbf{1})+(\mathbf{4}, \mathbf{1}, \mathbf{2})
\end{aligned}
$$

They are candidates for matter fermion generations and arise only from the twisted sectors

$$
\begin{aligned}
B_{p_{1} q_{1} r_{1} s_{1}=}^{1}= & S+b_{1}+p_{1} e_{3}+q_{1} e_{4}+r_{1} e_{5}+s_{1} e_{6} \\
= & \left\{\psi^{\mu}, x^{12},\left(y_{3} \bar{y}_{3}\right)^{1-p_{1}},\left(y_{4} \bar{y}_{4}\right)^{1-q_{1}},\left(y_{5} \bar{y}_{5}\right)^{1-r_{1}},\left(y_{6} \bar{y}_{6}\right)^{1-s_{1}},\right. \\
& \left.\left(\omega_{3} \bar{\omega}_{3}\right)^{p_{1}},\left(\omega_{4} \bar{\omega}_{4}\right)^{q_{1}},\left(\omega_{5} \bar{\omega}_{5}\right)^{r_{1}},\left(\omega_{6} \bar{\omega}_{6}\right)^{s_{1}}, \bar{\eta}^{1}, \bar{\psi}^{1, \ldots, 5}\right\} \\
B_{m_{2} n_{2} r_{2} s_{2}=}^{2}= & S+b_{2}+m_{2} e_{1}+n_{2} e_{2}+r_{2} e_{5}+s_{2} e_{6} \\
= & \left\{\psi^{\mu}, x^{34},\left(y_{1} \bar{y}_{1}\right)^{1-m_{2}},\left(y_{2} \bar{y}_{2}\right)^{1-n_{2}},\left(y_{5} \bar{y}_{5}\right)^{1-r_{2}},\left(y_{6} \bar{y}_{6}\right)^{1-s_{2}},\right. \\
& \left.\left(\omega_{1} \bar{\omega}_{2}\right)^{m_{2}},\left(\omega_{3} \bar{\omega}_{3}\right)^{n_{2}},\left(\omega_{5} \bar{\omega}_{5}\right)^{r_{2}},\left(\omega_{6} \bar{\omega}_{6}\right)^{s_{2}}, \bar{\eta}^{2}, \bar{\psi}^{1, \ldots, 5}\right\} \\
B_{m_{3} n_{3} p_{3} q_{3}=}^{3}= & S+b_{3}+m_{3} e_{1}+n_{3} e_{2}+p_{3} e_{3}+q_{3} e_{4} \\
= & \left\{\psi^{\mu}, x^{56},\left(y_{1} \bar{y}_{1}\right)^{1-m_{3}},\left(y_{2} \bar{y}_{2}\right)^{1-n_{3}},\left(y_{3} \bar{y}_{3}\right)^{1-p_{3}},\left(y_{4} \bar{y}_{4}\right)^{1-q_{3}},\right. \\
& \left.\left(\omega_{1} \bar{\omega}_{1}\right)^{m_{3}},\left(\omega_{2} \bar{\omega}_{2}\right)^{n_{3}},\left(\omega_{3} \bar{\omega}_{3}\right)^{p_{3}},\left(\omega_{4} \bar{\omega}_{4}\right)^{q_{3}}, \bar{\eta}^{3}, \bar{\psi}^{1, \ldots, 5}\right\}
\end{aligned}
$$

together with their supersymmetric partners from $S+B_{p_{1}, q_{1}, r_{1}, s_{1}}^{1}, S+B_{m_{2}, n_{2}, r_{2}, s_{2}}^{2}$ and $S+B_{m_{3}, n_{3}, p_{3}, q_{3}}^{3}$, where the upper index $I=1,2,3$ indicates the orbifold plane, $b_{3}=x+b_{1}+b_{2}$ and $m_{i}, n_{i}, p_{i}, q_{i}, r_{i}, s_{i}=$ 0,1 .

- States belonging to $S O(10)$ vectorials not charged under $S U(2)^{4} \times S O(8)$. Higgs doublets are accommodated in vectorial $S O(10)$ matter representations that arise from the sectors $V_{p q r s}^{I}=S+x+B_{p q r s}^{I}, I=1,2,3$ together with their superpartners from $S+V_{p q r s}^{I}$. The vectorial decomposition under $S O(10) \supset S U(4) \times S U(2)_{L} \times S U(2)_{R}$ is

$$
\mathbf{1 0}=(\mathbf{6}, \mathbf{1}, \mathbf{1})+(\mathbf{1}, \mathbf{2}, \mathbf{2})
$$

Three additional pairs of $(\mathbf{6}, \mathbf{1}, \mathbf{1})$ matter multiplets arise from the untwisted sector.

- Matter states charged under $S U(2)^{4} \times S O(8)$ but not under the Pati-Salam gauge symmetry. These arise from $(S)+B_{p q r s}^{I}+x+z_{1}, I=1,2,3$ and $B_{p q r s}^{I}+x+z_{2}, I=1,2,3$ and transform as hidden $S U(2) \times S U(2)$ bi-doublets or $S O(8)$ spinorials/vectorials. 


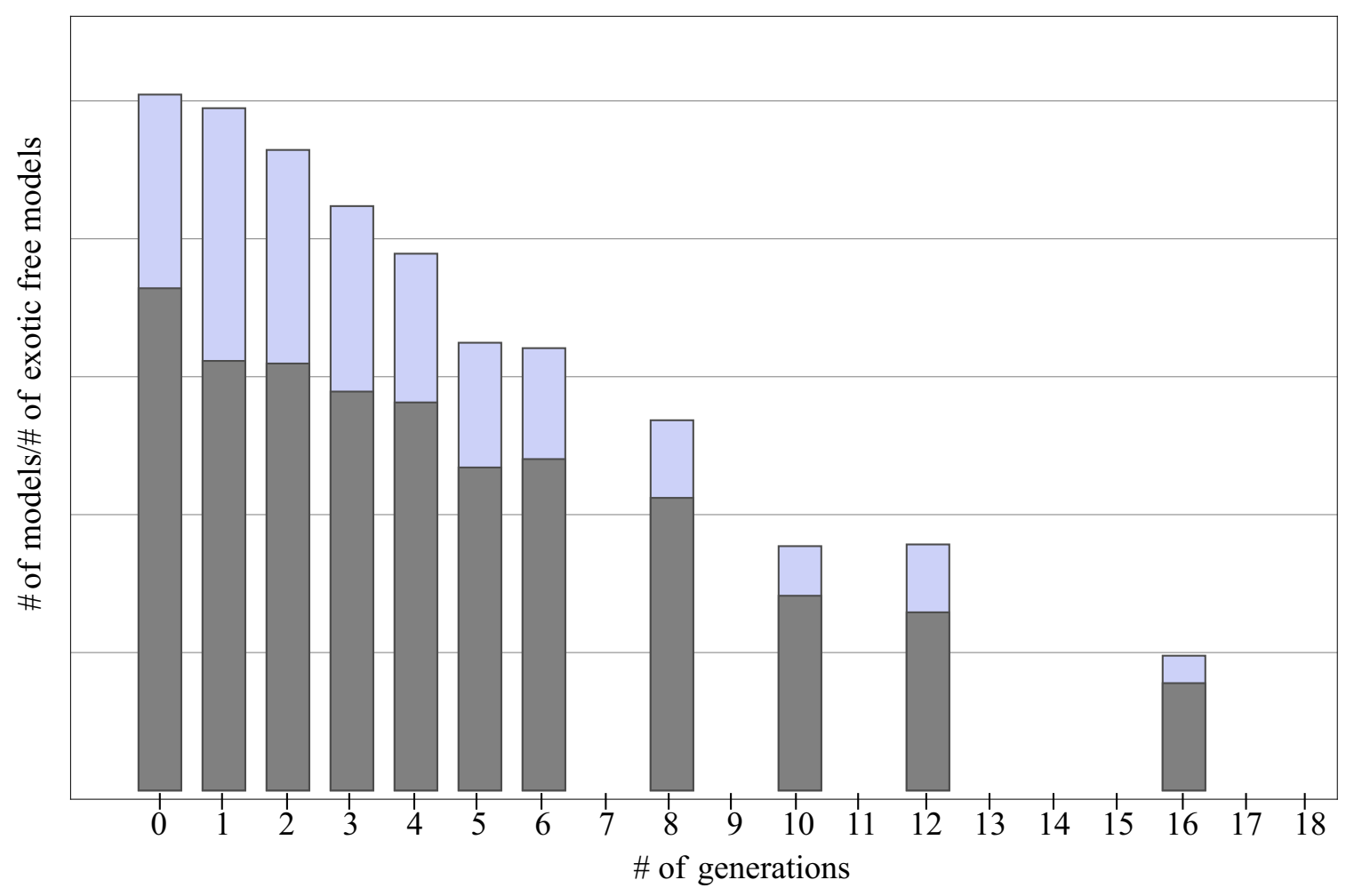

Figure 1: Total number of models and exotic free models (gray) in a random sample of $10^{11}$ Pati-Salam configurations.

- Mixed matter states that are charged under both the observable and the hidden sector. These are exotic fractionally charged states and arise from the sectors $(S)+B_{p q r s}^{I}+x, I=1,2,3$ and $B_{p q r s}^{I}+x+\alpha, I=1,2,3$

- Non-abelian gauge group singlet states charged only under $U(1)^{3}$, arising from both the twisted and the untwisted sectors.

After some tedious algebra we can derive analytic formulae for the multiplicities and the chiralities (when applicable) of each of the above matter spectrum representations, expressed in terms of the independent GGSO phases. These formulae can be evaluated using a fast computer algorithm and explicitly scan model spectra, in order either to classify them, or to identify models whose spectrum meets certain phenomenological criteria. To reduce computer time we can statistically sample the space of possible configurations by generating random sets of GGSO coefficients. A characteristic

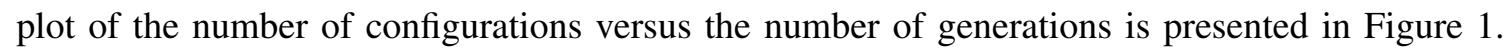
Detailed results are given in [ए]].

The phenomenological criteria employed in [ए] $]$ include

- Impose 3 complete fermion generations $3 \times[(\mathbf{4}, \mathbf{2}, \mathbf{1})+(\overline{\mathbf{4}}, \mathbf{1}, \mathbf{2})]$ and at least one pair of PS breaking Higgs $(\mathbf{4}, \mathbf{1}, \mathbf{2})+(\overline{\mathbf{4}}, \mathbf{1}, \mathbf{2})$.

- Existence of massless Higgs doublets $(\mathbf{1}, \mathbf{2}, \mathbf{2})$. 
- No massless fractionally charged exotics. Existence of these states in the spectrum of a string model is a generic property [ $\mathbb{W}]$, however their presence is hard to reconcile with standard cosmology [15]. As shown in Figure $\mathbb{W}$ this class contains a plethora of massless exotic free models.

A statistical computer scan over a sample of $10^{11}$ out of $10^{15}$ configurations shows that around one to a million $\left(1: 10^{6}\right)$ satisfy all these phenomenological constraints.

Additional phenomenological constraints need to be imposed in order to further narrow the space of candidate models. An important selection criterion is the existence of the top quark mass term at the tri-level superpotential. Apart from the gauge part, this coupling involves an Ising type correlation function that needs to be evaluated for a generic configuration. A detailed calculation shows that this requirement can be used to fix 12 extra GGSO phases [[13]

$$
\begin{array}{r}
c\left[\begin{array}{l}
e_{1} \\
b_{1}
\end{array}\right]=c\left[\begin{array}{l}
e_{2} \\
b_{1}
\end{array}\right]=c\left[\begin{array}{l}
e_{3} \\
b_{2}
\end{array}\right]=c\left[\begin{array}{l}
e_{4} \\
b_{2}
\end{array}\right]=c\left[\begin{array}{l}
z_{1} \\
b_{1}
\end{array}\right]=c\left[\begin{array}{l}
z_{1} \\
b_{2}
\end{array}\right]=0 \\
c\left[\begin{array}{l}
z_{2} \\
b_{1}
\end{array}\right]=c\left[\begin{array}{l}
z_{2} \\
b_{2}
\end{array}\right]=c\left[\begin{array}{l}
b_{1} \\
\alpha
\end{array}\right]=-c\left[\begin{array}{l}
b_{2} \\
\alpha
\end{array}\right]=+1 \\
c\left[\begin{array}{l}
e_{5} \\
b_{1}
\end{array}\right]=c\left[\begin{array}{l}
e_{5} \\
b_{2}
\end{array}\right], c\left[\begin{array}{l}
e_{6} \\
b_{1}
\end{array}\right]=c\left[\begin{array}{l}
e_{6} \\
b_{2}
\end{array}\right]
\end{array}
$$

The number of acceptable models is significantly reduced to a few million that can be fully classified with the help of a computer program. Removing redundancies we end up with a few hundred distinct models that meet all phenomenological criteria.

\section{A Pati-Salam exemplary Model}

Our classification method has revealed a subclass of Pati-Salam vacua, that satisfy all selection criteria and are thus good candidates for low energy models. However, compatibility with Minimal Supersymmetric Standard Model requires a detailed analysis, including the calculation of the superpotential and the solution of F- and (anomalous) D-flatness conditions. One has further to check weather the flat directions are compatible with the breaking of the PS gauge symmetry to the MMSM gauge group and also keep at least some of the Higgs doublets light. To this end, a concrete model has been constructed in [[4]]. The model is defined by the basis ([2.3]) and the 


\begin{tabular}{|l|c|c|}
\hline field symbol & $S U(4) \times S U(2)^{2}$ & $U(1)^{3}$ charges \\
\hline$F_{1 L}, F_{2 L}, F_{3 L}$ & $(\mathbf{4}, \mathbf{2}, \mathbf{1})$ & $\left(0,-\frac{1}{2}, 0\right),\left(0,0,-\frac{1}{2}\right),\left(0,0,+\frac{1}{2}\right)$ \\
\hline $\bar{F}_{1 R}, \bar{F}_{2 R}, \bar{F}_{3 R}, \bar{F}_{4 R}$ & $(\overline{\mathbf{4}}, \mathbf{1}, \mathbf{2})$ & $\left(0,-\frac{1}{2}, 0\right),\left(\frac{1}{2}, 0,0\right),\left(0,0, \frac{1}{2}\right),\left(0,0,-\frac{1}{2}\right)$ \\
\hline$h_{1}, h_{2}, h_{3}$ & $(\mathbf{1}, \mathbf{2}, \mathbf{2})$ & $\left(-\frac{1}{2}, 0,-\frac{1}{2}\right),\left(0, \frac{1}{2}, \frac{1}{2}\right),\left(0,-\frac{1}{2},-\frac{1}{2}\right)$ \\
\hline$D_{1}, \bar{D}_{1}, D_{2}, \bar{D}_{2}, D_{3}, \bar{D}_{3}, D_{4}$ & $(\mathbf{6}, \mathbf{1}, \mathbf{1})$ & $( \pm 1,0,0),(0, \pm 1,0),(0,0, \pm 1),\left(-\frac{1}{2}, 0,-\frac{1}{2}\right)$ \\
\hline$\Phi_{12}, \bar{\Phi}_{12}, \Phi_{13}, \bar{\Phi}_{13}$ & $(\mathbf{1}, \mathbf{1}, \mathbf{1})$ & $( \pm 1, \pm 1,0),( \pm 1,0, \pm 1)$ \\
\hline$\Phi_{12}^{-}, \bar{\Phi}_{12}^{-}, \Phi_{13}^{-}, \bar{\Phi}_{13}^{-}$ & $(\mathbf{1}, \mathbf{1}, \mathbf{1})$ & $(0, \pm 1, \pm 1),(0, \pm 1, \mp 1)$ \\
\hline$\Phi_{23}, \bar{\Phi}_{23}, \Phi_{23}^{-}, \bar{\Phi}_{23}^{-}$ & $(\mathbf{1}, \mathbf{1}, \mathbf{1})$ & $(0,0,0)$ \\
\hline$\Phi_{i}, i=1, \ldots, 6$ & $(\mathbf{1}, \mathbf{1}, \mathbf{1})$ & $\left( \pm \frac{1}{2}, \mp \frac{1}{2}, 0\right),\left( \pm \frac{1}{2}, \pm \frac{1}{2}, 0\right),\left( \pm \frac{1}{2}, 0, \mp \frac{1}{2}\right)$ \\
\hline$\zeta_{a}, \bar{\zeta}_{a}, a=1,2,3$ & $(\mathbf{1}, \mathbf{1}, \mathbf{1})$ & $\left( \pm \frac{1}{2}, 0, \mp \frac{1}{2}\right),\left(0, \pm \frac{1}{2}, \pm \frac{1}{2}\right),\left(0, \pm \frac{1}{2}, \pm \frac{1}{2}\right)$ \\
\hline$\zeta_{a}, \bar{\zeta}_{a}, a=4,5,6$ & $(\mathbf{1}, \mathbf{1}, \mathbf{1})$ & $\left( \pm \frac{1}{2}, 0, \mp \frac{1}{2}\right),\left(\frac{1}{2}, \frac{1}{2}, \pm 1\right)$ \\
\hline$\zeta_{7}, \bar{\zeta}_{7}, \chi_{+}, \chi_{-}$ & $(\mathbf{1}, \mathbf{1}, \mathbf{1})$ & \\
\hline
\end{tabular}

Table 1: Exemplary model "observable" sector matter spectrum and $S U(4) \times S U(2)_{L} \times S U(2)_{R} \times U(1)^{3}$ quantum numbers.

GGSO choice

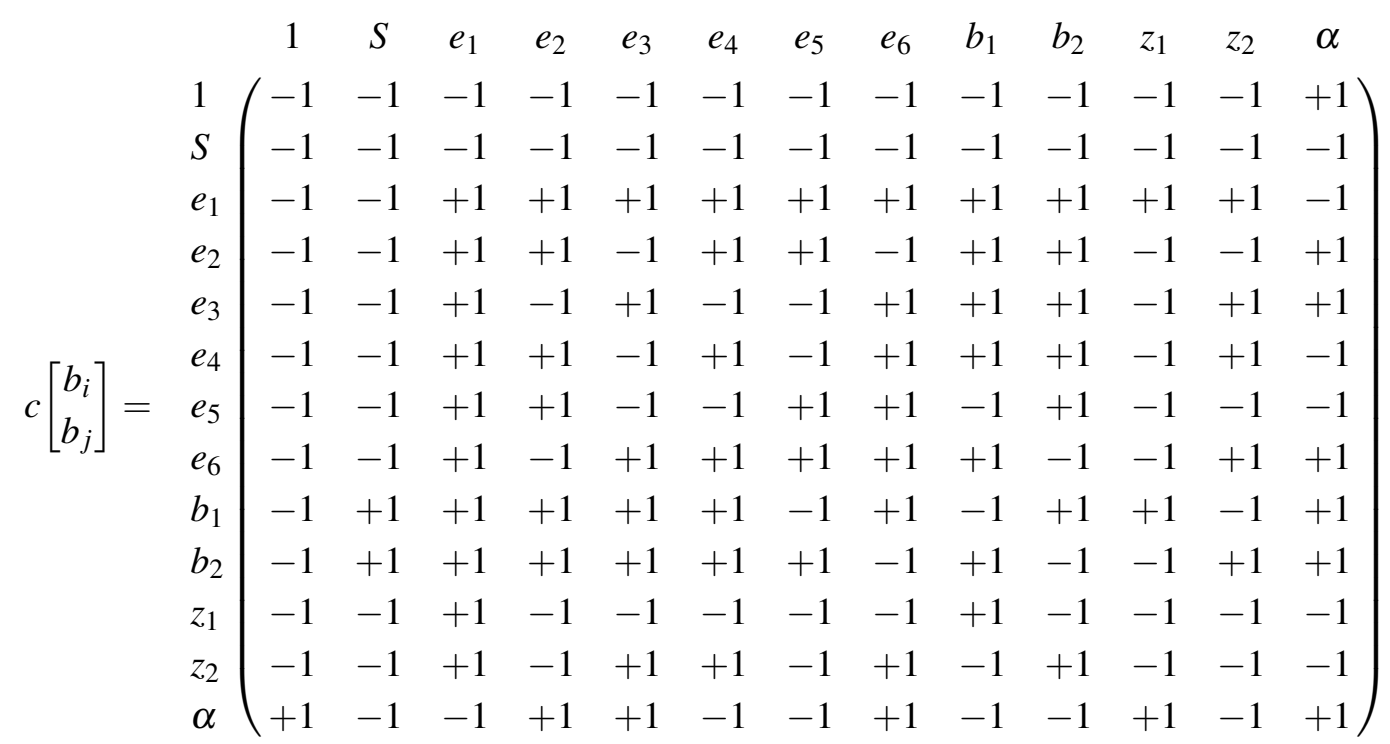

The full model spectrum is given in Tables $\square, \boldsymbol{D}$. The first contains the "observable sector" spectrum, that is states not charged under the $S U(2)^{4} \times S O(8)$ group factor. It consists of three $(\mathbf{4}, \mathbf{2}, \mathbf{1})+$ $(\overline{\mathbf{4}}, \mathbf{1}, \mathbf{2})$ multiplets that can accommodate the fermion generations, a pair of $(\mathbf{4}, \mathbf{1}, \mathbf{2}),(\overline{\mathbf{4}}, \mathbf{1}, \mathbf{2})$ representations that contain the Pati-Salam breaking Higg scalars, three pairs of Higgs bi-doublets $(\mathbf{1}, \mathbf{2}, \mathbf{2})$, seven pairs of extra triplets $(\mathbf{6}, \mathbf{1}, \mathbf{1})$ and a set of 34 non-abelian group singlets. The second table comprises the "hidden sector" states, that is states charged under the $S U(2)^{4} \times S O(8)$ group factor.

The full trilinear superpotential, $W$, can be calculated by a tedious but straightforward evaluation of the correlators of the associated vertex operators [ए]]

$$
W=g \sqrt{2}\left(W_{1}+W_{2}\right)
$$




\begin{tabular}{|l|c|c|}
\hline field symbol & $S U(2)^{4} \times S O(8)$ & $U(1)^{3}$ charges \\
\hline$H_{12}^{k}, k=1,2,3$ & $(\mathbf{2}, \mathbf{2}, \mathbf{1}, \mathbf{1}, \mathbf{1})$ & $\left(\mp \frac{1}{2},-\frac{1}{2}, 0\right),\left(\frac{1}{2}, 0,-\frac{1}{2}\right)$ \\
\hline$H_{13}^{k}, k=1,2,3$ & $(\mathbf{2}, \mathbf{1}, \mathbf{2}, \mathbf{1}, \mathbf{1})$ & $\left(-\frac{1}{2}, \mp \frac{1}{2}, 0\right),\left(\frac{1}{2},-\frac{1}{2}, 0\right)$ \\
\hline$H_{14}^{k}, k=1,2,3$ & $(\mathbf{2}, \mathbf{1}, \mathbf{1}, \mathbf{1}, \mathbf{1})$ & $\left(\frac{1}{2}, 0, \frac{1}{2}\right),\left(0, \pm \frac{1}{2}, \pm \frac{1}{2}\right)$ \\
\hline$H_{23}^{1}$ & $(\mathbf{1}, \mathbf{2}, \mathbf{2}, \mathbf{1}, \mathbf{1})$ & $\left(\frac{1}{2}, 0,+\frac{1}{2}\right)$ \\
\hline$H_{24}^{k}, k=1, \ldots, 5$ & $(\mathbf{1 , 2}, \mathbf{1}, \mathbf{2}, \mathbf{1})$ & $\left(-\frac{1}{2}, \pm \frac{1}{2}, 0\right),\left(-\frac{1}{2}, 0,-\frac{1}{2}\right),\left(0, \mp \frac{1}{2}, \pm \frac{1}{2}\right)$ \\
\hline$H_{34}^{k}, k=1, \ldots, 5$ & $(\mathbf{1}, \mathbf{1}, \mathbf{2}, \mathbf{2}, \mathbf{1})$ & $\left( \pm \frac{1}{2},-\frac{1}{2}, 0\right),\left(\frac{1}{2}, 0,-\frac{1}{2}\right),\left(0, \pm \frac{1}{2}, \pm \frac{1}{2}\right)$ \\
\hline$Z_{1}, Z_{3}$ & $\left(\mathbf{1}, \mathbf{1}, \mathbf{1}, \mathbf{1}, \mathbf{8}_{\mathbf{c}}\right)$ & $\left(-\frac{1}{2}, 0, \frac{1}{2}\right),\left(0,-\frac{1}{2}, \frac{1}{2}\right)$ \\
\hline$Z_{2}, Z_{4}$ & $\left(\mathbf{1}, \mathbf{1}, \mathbf{1}, \mathbf{1}, \mathbf{8}_{\mathbf{s}}\right)$ & $\left(0,-\frac{1}{2},-\frac{1}{2}\right),\left(0, \frac{1}{2},-\frac{1}{2}\right)$ \\
\hline
\end{tabular}

Table 2: Exemplary model "hidden" sector matter spectrum and $S U(2)^{2} \times S O(8) \times U(1)^{3}$ quantum numbers.

where

$$
\begin{aligned}
W_{1}= & \bar{F}_{2 R} F_{3 L} h_{1}+h_{1} h_{1} \Phi_{13}+h_{3} h_{3} \Phi_{23}+h_{2} h_{2} \bar{\Phi}_{23}+\frac{1}{\sqrt{2}} h_{1} h_{2} \zeta_{1}+D_{1} D_{2} \bar{\Phi}_{12} \\
& +\bar{D}_{1} D_{2} \Phi_{12}^{-}+D_{1} \bar{D}_{2} \bar{\Phi}_{12}^{-}+\bar{D}_{1} \bar{D}_{2} \Phi_{12}+D_{1} D_{3} \bar{\Phi}_{13}+\bar{D}_{1} D_{3} \Phi_{13}^{-}+D_{1} \bar{D}_{3} \bar{\Phi}_{13}^{-} \\
& +\bar{D}_{1} \bar{D}_{3} \Phi_{13}+D_{2} D_{3} \bar{\Phi}_{23}+\bar{D}_{2} D_{3} \Phi_{23}^{-}+D_{2} \bar{D}_{3} \bar{\Phi}_{23}^{-}+\bar{D}_{2} \bar{D}_{3} \Phi_{23} \\
& +D_{1} F_{1 R} F_{1 R}+\bar{D}_{1} \bar{F}_{2 R} \bar{F}_{2 R}+D_{2}\left(\bar{F}_{1 R} \bar{F}_{1 R}+F_{1 L} F_{1 L}\right)+D_{3}\left(\bar{F}_{4 R} \bar{F}_{4 R}+F_{2 L} F_{2 L}\right) \\
& +\bar{D}_{3}\left(\bar{F}_{3 R} \bar{F}_{3 R}+F_{3 L} F_{3 L}\right)+D_{4}\left(\bar{F}_{2 R} \bar{F}_{3 R}+D_{2} \chi-+\bar{D}_{2} \chi_{+}+D_{4} \Phi_{13}\right) \\
& +\bar{\Phi}_{13} \chi-\chi++\Phi_{23} \bar{\Phi}_{12} \Phi_{13}^{-}+\Phi_{13} \bar{\Phi}_{12} \Phi_{23}^{-}+\Phi_{23} \bar{\Phi}_{13} \Phi_{12}^{-}+\Phi_{12}^{-} \Phi_{23}^{-} \bar{\Phi}_{13}^{-} \\
& +\Phi_{13} \bar{\Phi}_{23} \bar{\Phi}_{12}^{-}+\Phi_{12} \bar{\Phi}_{23} \bar{\Phi}_{13}^{-}+\Phi_{13}^{-} \bar{\Phi}_{12}^{-} \bar{\Phi}_{23}^{-}+\Phi_{12} \bar{\Phi}_{13} \bar{\Phi}_{23}^{-} \\
& +\zeta_{1}^{2} \bar{\Phi}_{12}^{-}+\bar{\zeta}_{1}^{2} \Phi_{12}^{-}+\left(\zeta_{3}^{2}+\zeta_{4}^{2}+\zeta_{7}^{2}\right) \bar{\Phi}_{13}^{-}+\left(\bar{\zeta}_{3}^{2}+\bar{\zeta}_{4}^{2}+\bar{\zeta}_{7}^{2}\right) \Phi_{13}^{-} \\
& +\frac{1}{2} \bar{\zeta}_{2} \bar{\zeta}_{5} \chi++\zeta_{2}^{2} \bar{\Phi}_{12}+\left(\zeta_{5}^{2}+\zeta_{6}^{2}\right) \bar{\Phi}_{23}+\Phi_{12} \bar{\zeta}_{2}^{2}+\Phi_{5}\left(\zeta_{1} \bar{\zeta}_{1}+\zeta_{2} \bar{\zeta}_{2}\right) \\
& +\Phi_{2}\left(\zeta_{5} \bar{\zeta}_{5}+\zeta_{6} \bar{\zeta}_{6}\right)+\Phi_{23}\left(\bar{\zeta}_{5}^{2}+\bar{\zeta}_{6}^{2}\right)+\Phi_{4} \zeta_{7} \bar{\zeta}_{7}+\frac{1}{\sqrt{2}} \zeta_{4} \zeta_{5} \bar{\zeta}_{2}+\frac{1}{\sqrt{2}} \zeta_{2} \bar{\zeta}_{4} \bar{\zeta}_{5}
\end{aligned}
$$

and

$$
\begin{aligned}
W_{2}= & \bar{\Phi}_{23}\left\{\left(H_{34}^{4}\right)^{2}+\left(H_{14}^{2}\right)^{2}\right\}+\bar{\Phi}_{12}^{-}\left\{\left(H_{12}^{4}\right)^{2}+\left(H_{34}^{1}\right)^{2}\right\}+\bar{\Phi}_{13}^{-}\left\{\left(H_{12}^{3}\right)^{2}+\left(H_{34}^{2}\right)^{2}\right\} \\
& +\Phi_{12}^{-}\left\{\left(H_{13}^{2}\right)^{2}+\left(H_{24}^{1}\right)^{2}\right\}+\Phi_{12}\left\{\left(H_{12}^{1}\right)^{2}+\left(H_{13}^{1}\right)^{2}+\left(H_{24}^{2}\right)^{2}+\left(H_{34}^{2}\right)^{2}\right\}+\Phi_{13}\left\{\left(H_{13}^{3}\right)^{2}+\left(H_{24}^{3}\right)^{2}\right\} \\
& +\bar{\Phi}_{23}^{-} Z_{5}^{2}+\Phi_{13}^{-} Z_{1}^{2}+\Phi_{23}^{-} Z_{3}^{2}+\bar{\Phi}_{23}^{-}\left(H_{24}^{5}\right)^{2}+\Phi_{23}^{-}\left(H_{24}^{4}\right)^{2}+\Phi_{23}\left\{\left(H_{34}^{5}\right)^{2}+\left(H_{14}^{3}\right)^{2}\right\} \\
& +\frac{1}{\sqrt{2}} \zeta_{6}\left\{H_{12}^{1} H_{12}^{3}+H_{34}^{2} H_{34}^{3}\right\}+\frac{1}{\sqrt{2}} \zeta_{2} H_{24}^{3} H_{24}^{4}+\frac{1}{\sqrt{2}} \zeta_{7} H_{24}^{1} H_{24}^{4}+\frac{1}{\sqrt{2}} \bar{\zeta}_{1} H_{14}^{1} H_{14}^{3}+\frac{1}{\sqrt{2}} \bar{\zeta}_{2} H_{34}^{3} H_{34}^{4} \\
& +\chi+H_{34}^{2} H_{34}^{5}+H_{12}^{2} H_{14}^{2} H_{24}^{3}+H_{12}^{1} H_{14}^{1} H_{24}^{5}+H_{34}^{1} H_{13}^{3} H_{14}^{2}+H_{34}^{2} H_{24}^{5} H_{23}^{1}+\frac{1}{\sqrt{2}} \zeta_{1} Z_{1} Z_{5} \\
& +\bar{\Phi}_{23}^{-} Z_{5}^{2}+\Phi_{13}^{-} Z_{1}^{2}+\Phi_{23}^{-} Z_{3}^{2}+\Phi_{23}\left\{Z_{2}^{2}+Z_{4}^{2}\right\}
\end{aligned}
$$

The F-flatness conditions are given by

$$
F_{i}=\frac{\partial W}{\partial \phi_{i}}=0
$$


where $\phi_{i}$ runs over all fields in the model. Assuming zero vevs for the following fields

$$
\left\langle\bar{F}_{2 R}\right\rangle=\left\langle F_{3 L}\right\rangle=\left\langle D_{a}\right\rangle=\left\langle\bar{D}_{a}\right\rangle=\left\langle h_{i}\right\rangle=\left\langle Z_{i}\right\rangle=\left\langle H_{k l}^{m}\right\rangle=0
$$

we obtain a set of 31 equations

$$
\begin{aligned}
& \Phi_{2}: \zeta_{5} \bar{\zeta}_{5}+\zeta_{6} \bar{\zeta}_{6}=0 \\
& \Phi_{4}: \zeta_{7} \bar{\zeta}_{7}=0 \\
& \Phi_{5}: \zeta_{1} \bar{\zeta}_{1}+\zeta_{2} \bar{\zeta}_{2}=0 \\
& \Phi_{23}: \bar{\Phi}_{12} \Phi_{13}^{-}+\bar{\Phi}_{13} \Phi_{12}^{-}+\bar{\zeta}_{5}^{2}+\bar{\zeta}_{6}^{2}=0 \\
& \bar{\Phi}_{23}: \Phi_{13} \bar{\Phi}_{12}^{-}+\Phi_{12} \bar{\Phi}_{13}^{-}+\zeta_{5}^{2}+\zeta_{6}^{2}=0 \\
& \Phi_{23}^{-}: \Phi_{12}^{-} \bar{\Phi}_{13}^{-}+\Phi_{13} \bar{\Phi}_{12}=0 \\
& \bar{\Phi}_{23}^{-}: \Phi_{13}^{-} \bar{\Phi}_{12}^{-}+\Phi_{12} \bar{\Phi}_{13}=0 \\
& \Phi_{13}: \bar{\Phi}_{12} \Phi_{23}^{-}+\bar{\Phi}_{23} \bar{\Phi}_{12}^{-}=0 \\
& \bar{\Phi}_{13}: \Phi_{12} \bar{\Phi}_{23}^{-}+\chi_{-} \chi_{+}+\Phi_{23} \Phi_{12}^{-}=0 \\
& \Phi_{13}^{-}: \bar{\Phi}_{12}^{-} \bar{\Phi}_{23}^{-}+\bar{\zeta}_{3}^{2}+\bar{\zeta}_{4}^{2}+\bar{\zeta}_{7}^{2}+\Phi_{23} \bar{\Phi}_{12}=0 \\
& \bar{\Phi}_{13}^{-}: \Phi_{12} \bar{\Phi}_{23}+\Phi_{12}^{-} \Phi_{23}^{-}+\zeta_{3}^{2}+\zeta_{4}^{2}+\zeta_{7}^{2}=0 \\
& \Phi_{12}: \bar{\Phi}_{23} \bar{\Phi}_{13}^{-}+\bar{\Phi}_{13} \bar{\Phi}_{23}^{-}+\bar{\zeta}_{2}^{2}=0 \\
& \bar{\Phi}_{12}: \Phi_{23} \Phi_{13}^{-}+\Phi_{13} \Phi_{23}^{-}+\zeta_{2}^{2}=0 \\
& \Phi_{12}^{-}: \Phi_{23}^{-} \bar{\Phi}_{13}^{-}+\bar{\zeta}_{1}^{2}+\Phi_{23} \bar{\Phi}_{13}=0 \\
& \bar{\Phi}_{12}^{-}: \Phi_{13}^{-} \bar{\Phi}_{23}^{-}+\Phi_{13} \bar{\Phi}_{23}+\zeta_{1}^{2}=0 \\
& \zeta_{1}: 2 \zeta_{1} \bar{\Phi}_{12}^{-}+\Phi_{5} \bar{\zeta}_{1}=0 \\
& \bar{\zeta}_{1}: 2 \bar{\zeta}_{1} \Phi_{12}^{-}+\zeta_{1} \Phi_{5}=0 \\
& \zeta_{2}: 2 \zeta_{2} \bar{\Phi}_{12}+\Phi_{5} \bar{\zeta}_{2}+\frac{\bar{\zeta}_{4} \bar{\zeta}_{5}}{\sqrt{2}}=0 \\
& \bar{\zeta}_{2}: \frac{1}{2} \bar{\zeta}_{5} \chi_{+}+2 \Phi_{12} \bar{\zeta}_{2}+\zeta_{2} \Phi_{5}+\frac{\zeta_{4} \zeta_{5}}{\sqrt{2}}=0 \\
& \zeta_{3}: 2 \zeta_{3} \bar{\Phi}_{13}^{-}=0 \\
& \bar{\zeta}_{3}: 2 \bar{\zeta}_{3} \Phi_{13}^{-}=0 \\
& \zeta_{4}: 2 \zeta_{4} \bar{\Phi}_{13}^{-}+\frac{\zeta_{5} \bar{\zeta}_{2}}{\sqrt{2}}=0 \\
& \bar{\zeta}_{4}: 2 \bar{\zeta}_{4} \Phi_{13}^{-}+\frac{\zeta_{2} \bar{\zeta}_{5}}{\sqrt{2}}=0 \\
& \zeta_{5}: 2 \zeta_{5} \bar{\Phi}_{23}+\Phi_{2} \bar{\zeta}_{5}+\frac{\zeta_{4} \bar{\zeta}_{2}}{\sqrt{2}}=0 \\
& \bar{\zeta}_{5}: \frac{1}{2} \bar{\zeta}_{2} \chi_{+}+2 \Phi_{23} \bar{\zeta}_{5}+\frac{\zeta_{2} \bar{\zeta}_{4}}{\sqrt{2}}+\zeta_{5} \Phi_{2}=0 \\
& \zeta_{6}: 2 \zeta_{6} \bar{\Phi}_{23}+\Phi_{2} \bar{\zeta}_{6}=0
\end{aligned}
$$




$$
\begin{aligned}
& \bar{\zeta}_{6}: 2 \Phi_{23} \bar{\zeta}_{6}+\zeta_{6} \Phi_{2}=0 \\
& \zeta_{7}: 2 \zeta_{7} \bar{\Phi}_{13}^{-}+\Phi_{4} \bar{\zeta}_{7}=0 \\
& \bar{\zeta}_{7}: 2 \Phi_{13}^{-} \bar{\zeta}_{7}+\zeta_{7} \Phi_{4}=0 \\
& \chi_{+}: \bar{\Phi}_{13} \chi_{-}+\frac{1}{2} \bar{\zeta}_{2} \bar{\zeta}_{5}=0 \\
& \chi_{-}: \bar{\Phi}_{13} \chi_{+}=0
\end{aligned}
$$

The electroweak Higgs doublets are accommodated in the PS bi-doublets $h_{1}, h_{2}, h_{3}$. Their mass matrix is

$$
M_{h} \sim h_{h_{2}} h_{1}\left(\begin{array}{ccc}
h_{1} & h_{2} & h_{3} \\
\phi_{13} & \frac{\zeta_{1}}{\sqrt{2}} & 0 \\
\frac{\zeta_{1}}{\sqrt{2}} & \bar{\Phi}_{23} & 0 \\
0 & 0 & \Phi_{23}
\end{array}\right)
$$

$h_{1}$ doublet participates in the top quark coupling, hence we have to keep it massless imposing

$$
\Phi_{13} \bar{\Phi}_{23}-\frac{\zeta_{1}^{2}}{2}=0
$$

An exact solution of all the above conditions (B.7)-(B.39) is

$$
\begin{gathered}
0=\Phi_{1}=\Phi_{2}=\chi_{+}=\chi_{-}=\zeta_{i}=\bar{\zeta}_{i}, i=3, \ldots, 7 \\
\Phi_{5}=-\frac{2 i}{\sqrt{3}} \frac{\bar{\Phi}_{12} \sqrt{\frac{\Phi_{13}^{-} \Phi_{23}^{-} \bar{\Phi}_{23}^{-}}{\bar{\Phi}_{13}^{-}}}}{\Phi_{23}}=\frac{\Phi_{23}^{-} \bar{\Phi}_{23}^{-}}{\bar{\Phi}_{23}} \quad, \quad \Phi_{13}=-\frac{\Phi_{13}^{-} \bar{\Phi}_{23}^{-}}{3 \bar{\Phi}_{23}} \\
\bar{\Phi}_{13}=-\frac{3 \bar{\Phi}_{23} \bar{\Phi}_{13}^{-}}{\bar{\Phi}_{23}^{-}} \quad, \quad \Phi_{12}=-\frac{\bar{\Phi}_{12} \Phi_{13}^{-} \Phi_{23}^{-} \bar{\Phi}_{23}^{-}}{3 \bar{\Phi}_{23}^{2} \bar{\Phi}_{13}^{-}} \\
\Phi_{12}^{-}=\frac{\bar{\Phi}_{12} \Phi_{13}^{-} \bar{\Phi}_{23}^{-}}{3 \bar{\Phi}_{23} \bar{\Phi}_{13}^{-}} \quad, \quad \bar{\Phi}_{12}^{-}=-\frac{\bar{\Phi}_{12} \Phi_{23}^{-}}{\bar{\Phi}_{23}} \\
\zeta_{1}=i \sqrt{\frac{2 \Phi_{13}^{-} \bar{\Phi}_{23}^{-}}{3}} \quad, \quad \bar{\zeta}_{1}=-\sqrt{2 \Phi_{23}^{-} \bar{\Phi}_{13}^{-}} \\
\zeta_{2}=i \sqrt{\frac{2 \Phi_{23}^{-} \Phi_{13}^{-} \bar{\Phi}_{23}^{-}}{3 \bar{\Phi}_{23}}} \quad, \quad \bar{\zeta}_{2}=\sqrt{2 \bar{\Phi}_{23} \bar{\Phi}_{13}^{-}}
\end{gathered}
$$

It can be expressed in terms of nine parameters $\left\{\Phi_{3}, \Phi_{4}, \Phi_{6}, \bar{\Phi}_{23}, \Phi_{23}^{-}, \bar{\Phi}_{23}^{-}, \Phi_{13}^{-}, \bar{\Phi}_{13}^{-}, \bar{\Phi}_{12}\right\}$.

The abelian $U(1)$ group factors turn out to be anomalous

$$
\operatorname{Tr} U(1)_{1}=-12, \operatorname{Tr} U(1)_{2}=-24, \operatorname{Tr} U(1)_{3}=-12
$$

The can be rewritten as two anomaly free linear combinations

$$
\begin{aligned}
& U(1)_{1}^{\prime}=U(1)_{1}-U(1)_{3} \\
& U(1)_{2}^{\prime}=U(1)_{1}-U(1)_{2}+U(1)_{3}
\end{aligned}
$$


and one anomalous one

$$
U(1)_{A}^{\prime}=U(1)_{1}+2 U(1)_{2}+U(1)_{3}, \operatorname{Tr} U(1)_{A}=-72
$$

They lead to a set of three non-trivial D-flatness equations [1[4] that can be used to fix three of our free parameters. Moreover, this solution has the advantage of rendering all additional MSSM triplets, accommodated $(\mathbf{6}, \mathbf{1}, \mathbf{1})$, superheavy leaving only the MSSM spectrum at low energies. The above demonstrate that models in this class are of particular phenomenological interest and deserve further analysis.

\section{References}

[1] see e.g.

L. Susskind, In *Carr, Bernard (ed.): Universe or multiverse?* 247-266 [hep-th/0302219];

M. R. Douglas, JHEP 0305 (2003) 046 [hep-th/0303194];

T. Banks, M. Dine and E. Gorbatov, JHEP 0408 (2004) 058 [hep-th/0309170].

[2] see eg:

T.P.T. Dijkstra, L. Huiszoon and A.N. Schellekens, Nucl. Phys. B 710(2005) 3;

B.S. Acharya, F. Denef and R. Valadro, JHEP 0506(2005)056;

F. Gmeiner, R. Blumenhagen, G. Honecker, D. Lust and T. Weigand, JHEP 0601 (2006) 004;

E. Kiritsis, M. Lennek and B. Schellekens, Nucl. Phys. B 829 (2010) 298.;

M. Maio and A. N. Schellekens, Nucl. Phys. B 848, 594 (2011);

B. Gato-Rivera and A. N. Schellekens, Nucl. Phys. B 847, 532 (2011); Nucl. Phys. B 846, 429 (2011).

M. Maio, "Permutation Orbifolds in Conformal Field Theories and String Theory," [arXiv:1111.0991 [hep-th]].

[3] D. Senechal, Phys. Rev. D 39 (1989) 3717;

K. R. Dienes, Phys. Rev. D 73 (2006) 106010;

O. Lebedev, H. P. Nilles, S. Ramos-Sanchez, M. Ratz and P. K. S. Vaudrevange, Phys. Lett. B 668 (2008) 331;

F. Gmeiner, R. Blumenhagen, G. Honecker, D. Lust and T. Weigand, JHEP 0601 (2006) 004;

H. P. Nilles, S. Ramos-Sanchez, P. K. S. Vaudrevange and A. Wingerter, Comput. Phys. Commun. 183, 1363 (2012) [arXiv:1110.5229 [hep-th]];

T. Renner, J. Greenwald, D. Moore and G. Cleaver, Int. J. Mod. Phys. A 26 (2011) 4451

[arXiv:1107.3138 [hep-ph]].

[4] L. B. Anderson, J. Gray, A. Lukas and E. Palti, Phys. Rev. D 84 (2011) 106005 [[arXiv:1106.4804 [hep-th]];

L. B. Anderson, J. Gray, A. Lukas and E. Palti, "Heterotic Line Bundle Standard Models," arXiv:1202.1757 [hep-th].

[5] A. Gregori, C. Kounnas and J. Rizos, Nucl. Phys. B549 (1999) 16.

[6] A. E. Faraggi, C. Kounnas, S. E. M. Nooij and J. Rizos, proceedings of 2nd International Conference on String Phenomenology 2003, Durham, England, 29 Jul - 4 Aug 2003, [arXiv:hep-th/0311058];

A. E. Faraggi, C. Kounnas, S. E. M. Nooij and J. Rizos, Nucl. Phys. B 695 (2004) 41;

A. E. Faraggi, C. Kounnas and J. Rizos, Phys. Lett. B 648 (2007) 84; Nucl. Phys. B 774 (2007) 208;

Nucl. Phys. B 799 (2008) 19;

T. Catelin-Jullien, A. E. Faraggi, C. Kounnas and J. Rizos, Nucl. Phys. B 812 (2009) 103. 
[7] I. Antoniadis, C. Bachas, and C. Kounnas, Nucl. Phys. B289 (1987) 87; H. Kawai, D.C. Lewellen, and S.H.-H. Tye, Nucl. Phys. B288 (1987) 1; I. Antoniadis and C. Bachas, Nucl. Phys. B298 (1988) 586.

[8] J. C. Pati and A. Salam, Phys. Rev. D 10 (1974) 275 [Erratum-ibid. D 11 (1975) 703].

[9] I. Antoniadis and G. K. Leontaris, Phys. Lett. B 216 (1989) 333;

I. Antoniadis, G. K. Leontaris and J. Rizos, Phys. Lett. B 245 (1990) 161;

G. K. Leontaris and J. Rizos, Nucl. Phys. B 554 (1999) 3 [hep-th/9901098].

[10] B. Assel, K. Christodoulides, Nucl. Phys. B 844 (2011) 365 [arXiv:1007.2268 [hep-th]]; J. Rizos, Fortsch. Phys. 58 (2010) 758 [arXiv:1003.0458 [hep-th]].

[11] A. N. Schellekens, Phys. Lett. B 237 (1990) 363.

[12] B. Assel, K. Christodoulides, A. E. Faraggi, C. Kounnas and J. Rizos, Phys. Lett. B 683 (2010) 306.

[13] J. Rizos, in preparation.

[14] K. Christodoulides, A. E. Faraggi and J. Rizos, Phys. Lett. B 702 (2011) 81 [arXiv:1104.2264 [hep-ph]].

[15] P. Langacker and G. Steigman, Phys. Rev. D 84, 065040 (2011) [arXiv:1107.3131 [hep-ph]].

[16] S. Kalara, J. L. Lopez and D. V. Nanopoulos, Phys. Lett. B 245 (1990) 421;

J. Rizos and K. Tamvakis, Phys. Lett. B 251 (1990) 369; Phys. Lett. B 262 (1991) 227;

A. E. Faraggi, Nucl. Phys. B 487 (1997) 55 [hep-ph/9601332]. 